\title{
Correction of Polarization Error in Scanned Array Weather Radar Antennas
}

\author{
C. Pang ${ }^{1,2}$, P. Hoogeboom ${ }^{2,3}$, H. Russchenberg ${ }^{2}$, T. Wang ${ }^{1}$, J. Dong ${ }^{1}$, X. Wang ${ }^{1}$ \\ ${ }^{1}$ College of Electronic Science and Engineering, National University of Defense Technology, Changsha, China \\ ${ }^{2}$ Department of GRS, Faculty of Civil Engineering and Geoscience, Delft University of Technology, Delft, The Netherlands \\ ${ }^{3}$ TNO, Den Haag, The Netherlands \\ Email: c.pang@tudelft.nl
}

\begin{abstract}
In this paper, the polarization error correction of dual-polarized planar scanned array weather radar in alternately transmitting and simultaneously receiving (ATSR) mode is analyzed. A method based on point correction and a method taking the complete array patterns into account are discussed. To analyze their performances, a linear error model is presented and a Monte-Carlo simulation procedure is developed. The simulation results show that, since only the information at the beam direction is used, the cross-polar measurements of point correction method degrade with the beam scanning while the second method can effectively overcome this deficiency.
\end{abstract}

\section{INTRODUCTION}

Recently, the weather radar community has paid much attention to polarization phased array radar due to its agile electronic beam steering capacity which has the potential to significantly advance weather observation [1]. It's desirable to form a very narrow beam because of the distributed characteristic of meteorological targets. Nevertheless, for a mechanically scanning weather radar, a narrow beam still has considerable impacts on cross-polar measurements [2][6]. Moreover, for a phased array weather radar, it's inevitable that the beam will be expanded while scanning, which means the impacts of limited beamwidth will be emphasized when the beam is off broadside. Theoretically, if we know complete information of the polarization phased array radar system, these impacts can be precisely corrected. However, in many situations it's difficult to know the complete information. In this paper, the correction methods in ATSR mode will be analyzed based on the array information we know.

\section{FORMULATION}

The polarization scattering matrix (PSM) of a single precipitation particle is defined as

$$
S=\left[\begin{array}{ll}
S_{h h} & S_{v h} \\
S_{v h} & S_{v v}
\end{array}\right]
$$

Here we assume that the precipitation particle is reciprocal. And the received voltages are [2]

$$
V=\left[\begin{array}{ll}
V_{h h} & V_{h v} \\
V_{v h} & V_{v v}
\end{array}\right]=R^{\mathrm{T}} \cdot S \cdot T
$$

where $R$ and $T$ are the reception and transmission patterns

$$
R=\left[\begin{array}{ll}
R_{h h} & R_{h v} \\
R_{v h} & R_{v v}
\end{array}\right], T=\left[\begin{array}{ll}
T_{h h} & T_{h v} \\
T_{v h} & T_{v v}
\end{array}\right]
$$

T means matrix transpose. Note that in (2) the terms related to gain and range are omitted since we only focus on the array patterns. Then, the received voltages of the precipitation are expressed as an integral

$$
V=\int_{\Omega} R^{\mathrm{T}} \cdot S \cdot T d \Omega
$$

where $\Omega$ is solid angle and $d \Omega=\sin \theta d \theta d \varphi$.

\section{POLARIZATION ERROR CORRECTION}

\section{A. Model of Array Patterns}

As shown in figure 1 , the array with $N_{\text {row }}$ rows and $N_{\text {col }}$ columns is placed on $y z$ plane. We assume the array has $90^{\circ}$ angular range in azimuth and $30^{\circ}$ in elevation. Thus, in figure $1, \varphi$ is from $-45^{\circ} \sim 45^{\circ}$ and $\theta$ is from $60^{\circ} \sim 90^{\circ}$. For the sake of simplicity, the array patterns are written as

$$
T(\theta, \varphi)=\mathbf{f}_{a v e}(\theta, \varphi) \sum_{m=1}^{N_{\text {row }}} \sum_{n=1}^{N_{c o l}} \mathrm{e}^{j k \mathbf{r}_{m n} \hat{\mathbf{r}}} X_{m n}\left(\theta_{S}, \varphi_{S}\right)
$$

$$
R(\theta, \varphi)=\mathbf{f}_{a v e}(\theta, \varphi) \sum_{m=1}^{N_{\text {row }}} \sum_{n=1}^{N_{c o l}} \mathrm{e}^{j k \mathbf{r}_{m n} \hat{\mathbf{r}}} Y_{m n}\left(\theta_{S}, \varphi_{S}\right)
$$

where $\mathbf{f}_{\text {ave }}(\theta, \varphi)$ is the average elementary pattern. $X_{m n}$ and $Y_{m n}$ are array weighting coefficients, which are chosen for particular beam forming purpose and already known. If we get $\mathbf{f}_{a v e}(\theta, \varphi)$, then $T(\theta, \varphi)$ and $R(\theta, \varphi)$ can be calculated. Further, $\mathbf{f}_{\text {ave }}(\theta, \varphi)$ can be expressed as

$$
\mathbf{f}_{a v e}(\theta, \varphi)=\left[\begin{array}{ll}
f_{h h}(\theta, \varphi) & f_{h v}(\theta, \varphi) \\
f_{v h}(\theta, \varphi) & f_{v v}(\theta, \varphi)
\end{array}\right]
$$




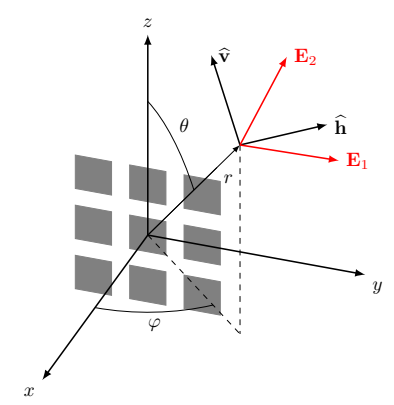

Fig. 1. Polarization distortion of dual-polarized planar array

\section{B. Point Correction}

The polarization distortion of dual-polarized array is shown in figure 1. $\mathbf{E}_{1}$ is the radiated electric field from $\mathrm{H}$ channel while $\mathbf{E}_{2}$ is from $\mathrm{V}$ channel. When the beam is off the broadside of the array, $\mathbf{E}_{1}$ and $\mathbf{E}_{2}$ are no longer orthogonal, which will introduce cross-polar errors that are not negligible. Hence the correction for polarization distortion is necessary, which can be written as written as [1]

$$
\widehat{V}=C_{R} \cdot V \cdot C_{T}
$$

where $C_{T}=\left(\left.T\left(\theta, \varphi, \theta_{S}, \varphi_{S}\right)\right|_{\theta=\theta_{S}, \varphi=\varphi_{S}}\right)^{-1}, C_{R}=$ $\left(\left.R^{\mathrm{T}}\left(\theta, \varphi, \theta_{S}, \varphi_{S}\right)\right|_{\theta=\theta_{S}, \varphi=\varphi_{S}}\right)^{-1}$ and $\left(\theta_{S}, \varphi_{S}\right)$ is the beam direction. (8) can be referred as "point correction", which means only the information at $\left(\theta_{S}, \varphi_{S}\right)$ is used. The performance of point correction will be discussed in the next section.

\section{Correction Based on Complete Patterns}

To take the complete array patterns into account, it's more convenient to apply the vector form. We define

$$
\mathbf{S}=\left[\begin{array}{l}
S_{h h} \\
S_{v h} \\
S_{v v}
\end{array}\right], \mathbf{V}=\left[\begin{array}{l}
V_{h h} \\
V_{v h} \\
V_{v v}
\end{array}\right]
$$

Then, we have

$$
\mathbf{V}=D \cdot \mathbf{S}
$$

where

$$
D=\left[\begin{array}{ccc}
T_{h h} R_{h h} & T_{h h} R_{v h}+T_{v h} R_{h h} & T_{v h} R_{v h} \\
T_{h h} R_{h v} & T_{h h} R_{v v}+T_{v h} R_{h v} & T_{v h} R_{v v} \\
T_{h v} R_{h v} & T_{h v} R_{v v}+T_{v v} R_{h v} & T_{v v} R_{v v}
\end{array}\right]
$$

The received voltage covariance matrix is defined as

$$
\mathbf{V} \cdot \mathbf{V}^{\mathrm{H}}=D \cdot \mathbf{S} \cdot(D \cdot \mathbf{S})^{\mathrm{H}}=D \cdot \mathbf{S} \cdot \mathbf{S}^{\mathrm{H}} \cdot D^{\mathrm{H}}
$$

where $\mathrm{H}$ means Hermitian Transpose. (12) can be expressed as the form of the product of matrix and vector [3]

$$
\mathbf{v}=M \cdot \mathbf{s}
$$

and

$$
M=D \otimes D^{*}
$$

where $\otimes$ denotes the Kronecker matrix product, * means complex conjugate, and $\mathbf{v}, \mathbf{s}$ are $9 \times 1$ vectors formed by stacking the rows of $\mathbf{V} \cdot \mathbf{V}^{\mathrm{H}}, \mathbf{S} \cdot \mathbf{S}^{\mathrm{H}}$, respectively. Then, the received voltage covariance vector of the precipitation is expressed as an integral

$$
\mathbf{v}=\left(\int_{\Omega} M d \Omega\right) \cdot\langle\mathbf{s}\rangle
$$

where $\langle\bullet\rangle$ means assemble average. Then we define

$$
P=\int_{\Omega} M d \Omega
$$

$P$ is a $9 \times 9$ matrix. In theory, if we know the transmission and reception patterns $R$ and $T$, including both amplitudes and phases, the intrinsic covariance matrix $\mathbf{S} \cdot \mathbf{S}^{\mathrm{H}}$ can be retrieved precisely through

$$
\langle\mathbf{s}\rangle=P^{-1} \cdot \mathbf{v}
$$

\section{Error Model}

The corrected voltage matrix can be expressed as

$$
\widehat{V}=\int_{\Omega} \widehat{R}^{\mathrm{T}} \cdot S \cdot \widehat{T} d \Omega
$$

where $\widehat{T}$ and $\widehat{R}$ are called corrected transmission and reception patterns

$$
\widehat{T}=T \cdot C_{T}=\left[\begin{array}{cc}
\widehat{T}_{h h} & \widehat{T}_{h v} \\
\widehat{T}_{v h} & \widehat{T}_{v v}
\end{array}\right]
$$

$$
\widehat{R}=R \cdot\left(C_{R}\right)^{\mathrm{T}}=\left[\begin{array}{ll}
\widehat{R}_{h h} & \widehat{R}_{h v} \\
\widehat{R}_{v h} & \widehat{R}_{v v}
\end{array}\right]
$$

$\widehat{T}$ and $\widehat{R}$ can be written as

$$
\begin{aligned}
& \widehat{T}=\left[\begin{array}{cc}
1+\varepsilon_{h h} & \varepsilon_{h v} \\
\varepsilon_{v h} & 1+\varepsilon_{v v}
\end{array}\right] \widehat{F}_{T} \\
& \widehat{R}=\left[\begin{array}{cc}
1+\varepsilon_{h h} & \varepsilon_{h v} \\
\varepsilon_{v h} & 1+\varepsilon_{v v}
\end{array}\right] \widehat{F}_{R}
\end{aligned}
$$

where $\varepsilon_{i j},(i, j=h, v)$ is the error term after the corrections and $\widehat{F}_{T}, \widehat{F}_{R}$ are the normalized array factors for transmission and reception, respectively. It's reasonable to approximate $\varepsilon_{i j}$ as a linear function of $\theta$ and $\varphi$, i.e., 
TABLE I. ARRAY PARAMETERS

\begin{tabular}{ll}
\hline Array size & $64 \times 64$ \\
Frequency $f_{c}$ & $3 \mathrm{GHz}$ \\
Element separation & $\lambda / 2$ \\
Transmission pattern weighting & uniform weighting \\
$\operatorname{Reception}$ pattern weighting & $-40 \mathrm{~dB}$ Taylor weighting \\
$\operatorname{Arg}\left(\alpha_{i j}\right)$ & $U(0,2 \pi)$ \\
$\operatorname{Arg}\left(\beta_{i j}\right)$ & $U(0,2 \pi)$ \\
$\operatorname{Arg}\left(\delta_{i j}\right)$ & $U(0,2 \pi)$ \\
&
\end{tabular}

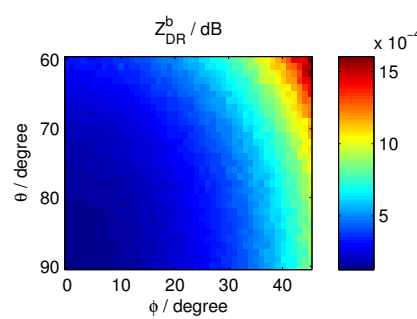

(a)

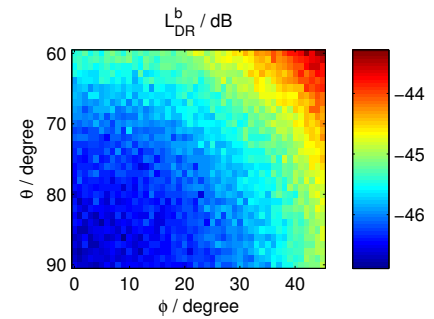

(b)
Fig. 2. Simulation results of point correction method with $\delta_{i j}=0$ and $\left|\alpha_{i j}\right|,\left|\beta_{i j}\right| \in U(0,0.5)$

$$
\varepsilon_{i j} \approx \alpha_{i j}\left(\theta-\theta_{S}\right)+\beta_{i j}\left(\varphi-\varphi_{S}\right)+\delta_{i j}
$$

where $\alpha_{i j}, \beta_{i j}$ and $\delta_{i j}$ are complex numbers. $\alpha_{i j}$ and $\beta_{i j}$ are the change rates of $\varepsilon_{i j}$ for $\theta$ and $\varphi$, respectively, which indicate the spatial polarization variations of the array. In other words, $\left|\alpha_{i j}\right|$ and $\left|\beta_{i j}\right|$ characterize the polarization purity in the neighborhood of $\left(\theta_{S}, \varphi_{S}\right)$. Taking the cross-placed dipoles for example, we have $\left|\alpha_{i j}\right|<2,\left|\beta_{i j}\right|<2$ in the scanning area.

If $C_{T}$ and $C_{R}$ are accurate, there should be $\varepsilon_{i j}\left(\theta_{S}, \varphi_{S}\right)=$ 0 , i.e., $\delta_{i j}=0$. However, due to some practical factors, $\delta_{i j}$ is not 0 , which indicates that $\delta_{i j}$ can be seen as a merit of correction. Hence, we call $\delta_{i j}$ is the polarization distortion correction error level.

\section{Simulation Results}

In order to analyze the performance of the point correction method, a Monte-Carlo simulation procedure based on the linear error model (23) is developed. Specifically, first, $\alpha_{i j}$, $\beta_{i j}$ and $\delta_{i j}$ are generated through a random number generator. Second, the corrected transmission and reception patterns $\widehat{T}$ and $\widehat{R}$ are calculated. Finally, the biased $Z_{D R}^{b}$ and $L_{D R}^{b}$ are obtained and the final $Z_{D R}^{b}$ and $L_{D R}^{b}$ at each direction are calculated by averaging the results of 1000 Monte-Carlo simulations. The simulation parameters are shown in table I in which $U(a, b)$ means uniform distribution in $[a, b]$ and $\operatorname{Arg}(z)$ represents the phase of complex number $z$.

Figure 2 7 show the simulation results of point correction method with different parameters.

Based on figure 2 7, we can conclude that the correction error, i.e., $\delta_{i j}$, is crucial for the polarimetric bias correction. It determines the measured average $Z_{D R}^{b}$ and the lower bound of $L_{D R}^{b}$ that an array system can achieve with correction error. In addition, the polarization variations of array antenna, i.e., $\alpha_{i j}$ and $\beta_{i j}$, do have considerable influences on the cross-polar component measurements, which indicates that an optimal

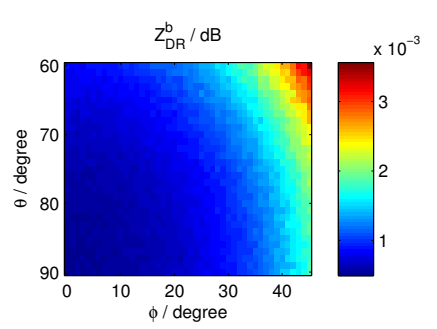

(a)

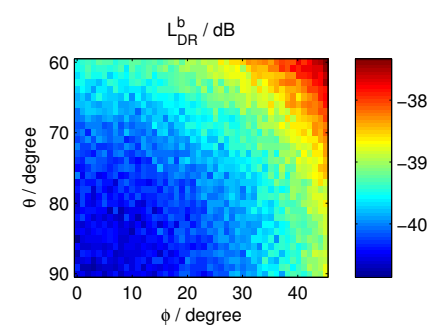

(b)
Fig. 3. Simulation results of point correction method with $\delta_{i j}=0$ and $\left|\alpha_{i j}\right|,\left|\beta_{i j}\right| \in U(0,1)$

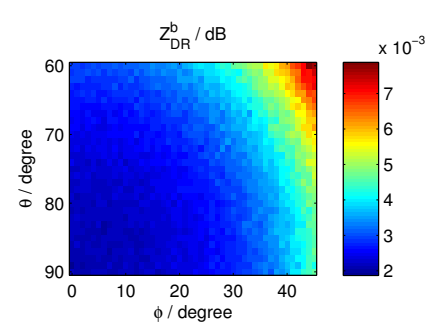

(a)

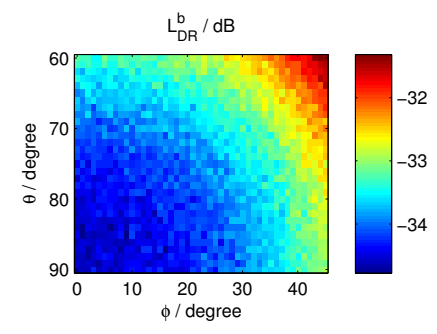

(b)
Fig. 4. Simulation results of point correction method with $\delta_{i j}=0$ and $\left|\alpha_{i j}\right|,\left|\beta_{i j}\right| \in U(0,2)$

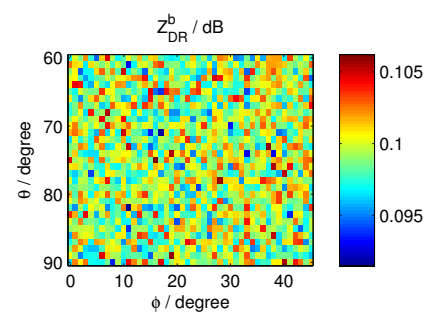

(a)

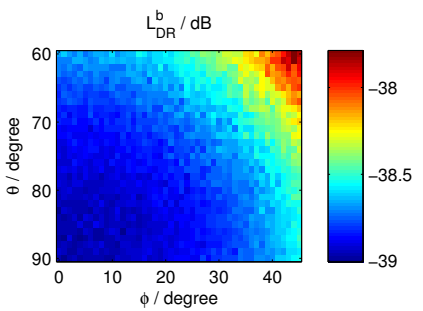

(b)
Fig. 5. Simulation results of point correction method with $\left|\delta_{i j}\right|=0.01$ and $\left|\alpha_{i j}\right|,\left|\beta_{i j}\right| \in U(0,0.5)$

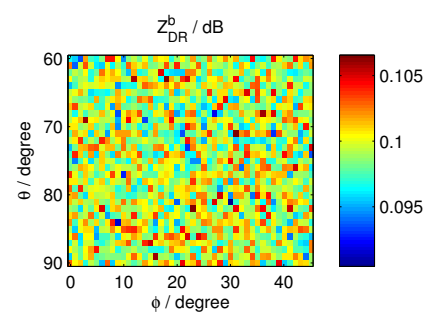

(a)

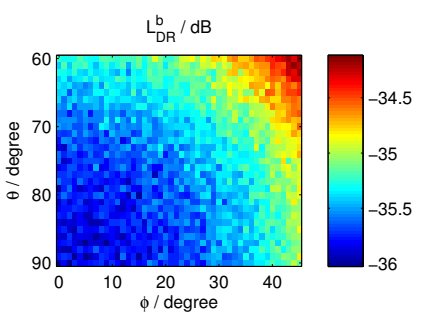

(b)
Fig. 6. Simulation results of point correction method with $\left|\delta_{i j}\right|=0.01$ and $\left|\alpha_{i j}\right|,\left|\beta_{i j}\right| \in U(0,1)$

design of array element and structure is beneficial to enhance the system sensitivity of cross-polar measurements.

The degradation of cross-polar measurements is because only the information at the beam direction is used and the polarization variations in the neighbourhood of beam direction are not considered. Actually, it's inevitable to measure the complete array patterns since we need to correct the biases for all directions. Therefore, it's possible to use the complete 


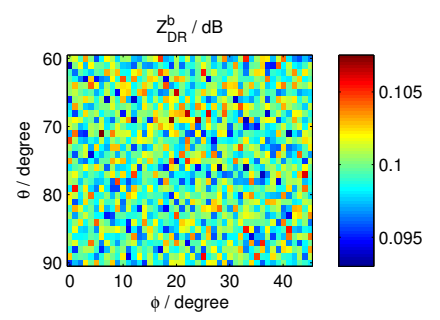

(a)

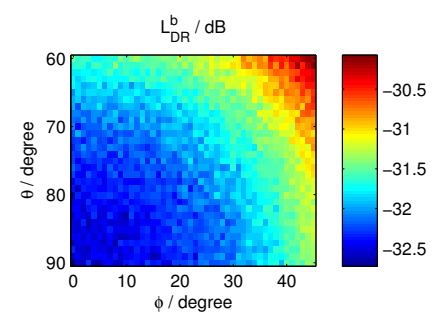

(b)

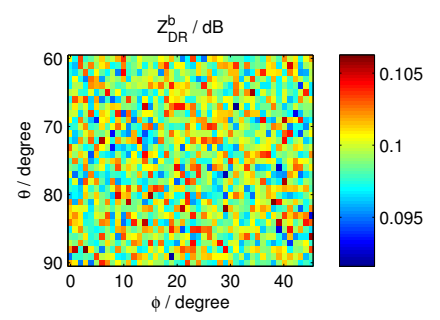

(a)

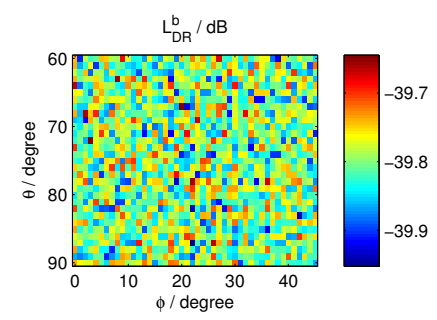

(b)

Fig. 7. Simulation results of point correction method with $\left|\delta_{i j}\right|=0.01$ and $\left|\alpha_{i j}\right|,\left|\beta_{i j}\right| \in U(0,2)$

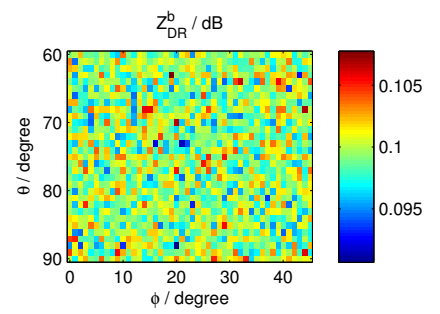

(a)

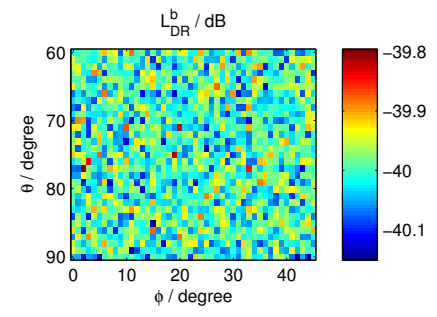

(b)

Fig. 8. Simulation results with $\left|\delta_{i j}\right|=0.01$ and $\left|\alpha_{i j}\right|=0,\left|\beta_{i j}\right|=0$

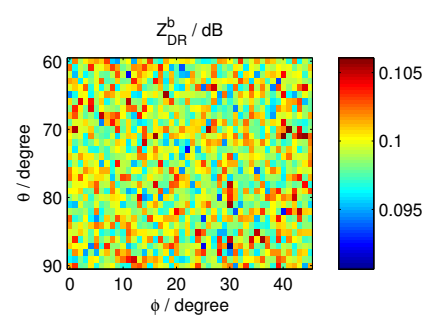

(a)

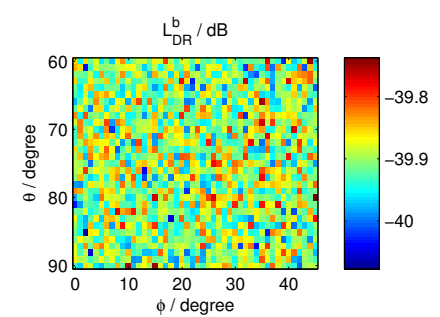

(b)

Fig. 9. Simulation results with $\left|\delta_{i j}\right|=0.01$ and $\left|\alpha_{i j}\right|,\left|\beta_{i j}\right| \in U(0,0.1)$

patterns for bias correction.

If the accurate array patterns are measured, there will be $\varepsilon_{i j}(\theta, \varphi)=0$, i.e., $\alpha_{i j}=0, \beta_{i j}=0, \delta_{i j}=0$. When considering the measurement errors of array patterns, we will have $\left|\delta_{i j}\right|>0$. Since the influences of polarization variations are almost compensated in the integration of $P,\left|\alpha_{i j}\right|$ and $\left|\beta_{i j}\right|$ will be close to 0 with the measurement errors.

Figure $8 \sim 10$ show the simulation results of the method based on complete patterns, in which we set $Z_{D R}=0 \mathrm{~dB}$, $L_{D R}=-\infty \mathrm{dB},\left|\delta_{i j}\right|=0.01$ and increase the variations of $\left|\alpha_{i j}\right|$ and $\left|\beta_{i j}\right|$ from 0 to 0.25 . From these figures we see that $L_{D R}^{b}$ is independent of the beam directions and they are all close to $-40 \mathrm{~dB}$, which demonstrates the good performance of the correction method based on complete patterns.

\section{Conclusions}

In this paper, two polarimetric bias correction methods for meteorological applications have been discussed and their performances have been analyzed. As mentioned above, the measurement errors of array patterns play an important role in the bias correction. Hence, the array pattern measurements

Fig. 10. Simulation results with $\left|\delta_{i j}\right|=0.01$ and $\left|\alpha_{i j}\right|,\left|\beta_{i j}\right| \in U(0,0.25)$

should be as accurate as possible so that the retrieved variables, such as $Z_{D R}$ and $L_{D R}$, are more reliable.

The point correction method is straightforward and easy to implement. However, its cross-polar measurement performance will degrade when the beam direction is away from the broadside because the polarization variations of array patterns in the neighbourhood of beam direction are not compensated. For a well-designed array having relatively small polarization variations, this degradation is slight and can be ignored when the intrinsic $L_{D R}$ of the precipitations is relatively large.

As to the correction method based on complete patterns, it can be seen as an extension of the point correction method, which requires more complicated computations. With the rapid development of computational hardware, which makes the high performance computing more affordable, the computation complexity of the method based on complete patterns is not a bottleneck. In some applications, the increased computations are worthwhile because of the improved cross-polar measurement performance.

\section{ACKNOWLEDGMENT}

This work was supported by the National Natural Science Foundation of China (CSNF) under Grant 61201330.

\section{REFERENCES}

[1] G. Zhang, R. J. Doviak, D. S. Zrnic, J. Crain, D. Staiman, and Y. AlRashid, "Phased array radar polarimetry for weather sensing: a theoretical formulation for bias corrections," Geoscience and Remote Sensing, IEEE Transactions on, vol. 47, no. 11, pp. 3679-3689, 2009.

[2] V. Chandrasekar and R. J. Keeler, "Antenna pattern analysis and measurements for multiparameter radars," Journal of Atmospheric and Oceanic Technology, vol. 10, pp. 674-683, 1993.

[3] D. N. Moisseev, C. M. Unal, H. W. Russchenberg, and L. P. Ligthart, "Improved polarimetric calibration for atmospheric radars," Journal of Atmospheric and Oceanic Technology, vol. 19, no. 12, pp. 1968-1977, 2002.

[4] Y. Wang and V. Chandrasekar, "Polarization isolation requirements for linear dual-polarization weather radar in simultaneous transmission mode of operation," Geoscience and Remote Sensing, IEEE Transactions on, vol. 44, no. 8, pp. 2019-2028, 2006.

[5] D. Zrnic, R. Doviak, G. Zhang, and A. Ryzhkov, "Bias in differential reflectivity due to cross coupling through the radiation patterns of polarimetric weather radars," Journal of Atmospheric and Oceanic Technology, vol. 27, no. 10, pp. 1624-1637, 2010.

[6] M. Galletti and D. S. Zrnic, "Bias in copolar correlation coefficient caused by antenna radiation patterns," Geoscience and Remote Sensing, IEEE Transactions on, vol. 49, no. 6, pp. 2274-2280, 2011. 